NASA Contractor Report 187518

ICASE Report No. 91-16

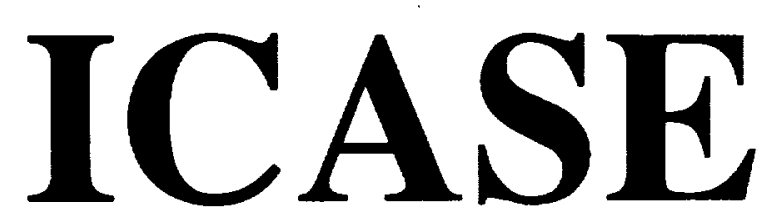

\title{
APPLICATION OF A NEW K- $\tau$ MODEL TO NEAR WALL TURBULENT FLOWS
}
S. Thangam
R. Abid
C. G. Speziale

Contract No. NAS1-18605

February 1991

Institute for Computer Applications in Science and Engineering NASA Langley Research Center

Hampton, Virginia 23665-5225

Operated by the Universities Space Research Association

\section{N/SA}

National Aeronautics and

Space Administration

Langley Research Center

Hampton, Virginia 23665-5225

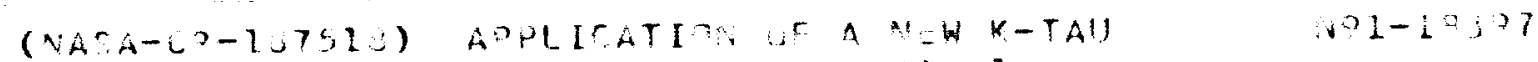

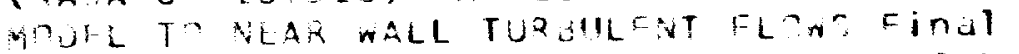

$$
\begin{aligned}
& \text { aport (ICASE) } 12 \mathrm{~F} \text { CSCL ?OO }
\end{aligned}
$$





\title{
Application of a New $K-\tau$ Model to Near Wall Turbulent Flows
}

\author{
S. Thangam ${ }^{\dagger \S}$ \\ ICASE, NASA Langley Research Center \\ Hampton, Virginia 23665 \\ R. Abid \\ Vigyan Research Associates \\ Hampton, Virginia 23665 \\ C.G. Speziale $\$$ \\ ICASE, NASA Langley Research Center \\ Hampton, Virginia 23665
}

\begin{abstract}
A recently developed $K$ - $\tau$ model for near wall turbulent flows is applied to two severe test cases. The turbulent flows considered include the incompressible flat plate boundary layer with adverse pressure gradients and incompressible flow past a backward facing step. Calculations are performed for this two-equation model using an anisotropic as well as isotropic eddy-viscosity. The model predictions are shown to compare quite favorably with experimental data.
\end{abstract}

$\dagger$ Permanent Address: Department of Mechanical Engineering, Stevens Institute of Technology, Hoboken, NJ 07030

$\S$ This research was supported by the National Aeronautics and Space Administration under NASA Contract No. NAS1-18605 while the first and the third authors (ST \& CGS) were in residence at the Institute for Computer Applications in Science and Engineering (ICASE), NASA Langley Research Center, Hampton, VA 23665. 



\section{Introduction}

Two-equation turbulence models have become increasingly popular for the calculation of practical aerodynamic flows which can have important applications in the design of advanced aircraft. The reason for this popularity is clear: two-equation models incorporate substantially more turbulence physics - and require less ad hoc empiricisms - than the older algebraic eddy-viscosity models without most of the added difficulties that arise from the computational implementation of second-order closure models. Among the existing two-equation models, the $K-\varepsilon$ model is probably the most popular; its successes and limitations have been fairly well documented [1,2]. One major difficulty with the $K-\varepsilon$ model that has yet to be fully resolved involves its application to near wall turbulent flows where inaccuracies and numerical stiffness problems can arise (see Patel, Rodi and Scheuerer [3] for an interesting discussion of these problems). Recently, Speziale, Abid and Anderson [4] developed a new $K$ - $\tau$ model for near wall turbulent flows wherein modeled transport equations are solved for the turbulent kinetic energy $K$ and the turbulent time scale $\tau$. While the use of the turbulent time scale had been proposed earlier within the context of two equation models (cf., Reynolds [5] and Zeierman and Wolfshtein [6]), none of these previous studies rendered a model that could be integrated directly to a solid boundary with the no-slip condition applied. Speziale et al. [4] demonstrated that their new $K-\tau$ model yielded excellent results - and was computationally robust - when integrated directly to the wall in an incompressible flat plate boundary layer with zero pressure gradient. The $K-\tau$ model alleviates the problem of the lack of natural boundary conditions for the dissipation rate in the $K-\varepsilon$ model since the turbulent time scale $\tau$ vanishes identically at a solid boundary.

The purpose of the present paper is to provide a more comprehensive testing and evaluation of the $K$ - $\tau$ model. Two test cases are chosen to evaluate the model: (i) the incompressible flat plate boundary layer with adverse pressure gradients, and (ii) incompressible flow past a backward facing step. Unlike in the previous study [4], the $K$ - $\tau$ model will be solved with an anisotropic as well as isotropic eddy viscosity. It has been demonstrated during the past decade that, in some cases, the predictions of two-equation models can be enhanced considerably by the use of an anisotropic eddy viscosity $[7,8]$. The results obtained from the $K-\tau$ model will be documented in detail and recommendations will be made for future research.

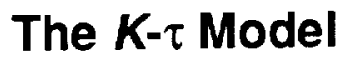

In the standard form of the $K$ - $\tau$ model, the Reynolds stress tensor $\tau_{i j} \equiv \overline{u_{i}^{\prime} u_{j}^{\prime}}$ (where $u_{i}^{\prime}$ is the fluctuating velocity) is of the form

$$
\tau_{i j}=\frac{2}{3} K \delta_{i j}-v_{T}\left(\frac{\partial \bar{u}_{i}}{\partial x_{j}}+\frac{\partial \bar{u}_{j}}{\partial x_{i}}\right)
$$

where the eddy viscosity is given by

$$
v_{T}=C_{\mu} f_{\mu} K \tau
$$


and $\bar{u}_{i}$ is the mean velocity, $K \equiv \frac{1}{2} \bar{u}_{i}^{\prime} u_{i}^{\prime}$ is the turbulent kinetic energy, $\varepsilon \equiv \vee \overline{\partial u_{i}^{\prime} / \partial x_{j} \partial u_{i}^{\prime} / \partial x_{j}}$ is the turbulent dissipation rate, $\tau \equiv K / \varepsilon$ is the turbulent time scale, and $C_{\mu}$ is a dimensionless constant that assumes a value of 0.09 . In (2), $f_{\mu}$ is a wall damping function that is needed for asymptotic consistency [4]; it is taken to be of the form

$$
f_{\mu}=\left(1+\frac{3.45}{\sqrt{R e_{t}}}\right) \tanh \left(\frac{y^{+}}{70}\right)
$$

where $R e_{t}=\frac{K \tau}{v}$ is the turbulence Reynolds number and $y^{+}=y u_{\tau} / v$ is the usual wall coordinate given that $u_{\tau}$ is shear velocity and $v$ is the kinematic viscosity. In an attached boundary layer, the shear velocity is usually taken to be $\sqrt{\tau_{0} / \rho}$ (where $\tau_{0}$ is the wall shear stress); for more complicated turbulent flows with separation, the shear velocity is assumed to be proportional to $K^{1 / 2}$. The wall damping function $f_{\mu}$ goes to unity sufficiently far from the wall. In the anisotropic version of the $K$ - $\tau$ model, nonlinear corrections are added to the eddy viscosity model as developed by Speziale [8]. This leads to the following Reynolds stress model

$$
\tau_{i j}=\frac{2}{3} K \delta_{i j}-2 C_{\mu} f_{\mu} K \tau \bar{S}_{i j}-4 C_{D} C_{\mu}^{2} f_{\mu}^{2} K \tau^{2}\left(\bar{S}_{i k} \bar{S}_{k j}-\frac{1}{3} \bar{S}_{k l} \bar{S}_{k l} \delta_{i j}+\stackrel{\circ}{S}_{i j}-\frac{1}{3} \stackrel{\circ}{S}_{k k} \delta_{i j}\right)
$$

where

$$
\begin{gathered}
\bar{S}_{i j}=\frac{1}{2}\left(\frac{\partial \bar{u}_{i}}{\partial x_{j}}+\frac{\partial \bar{u}_{j}}{\partial x_{i}}\right) \\
\stackrel{\circ}{S}_{i j}=\frac{\partial \bar{S}_{i j}}{\partial t}+\bar{u}_{k} \frac{\partial \bar{S}_{i j}}{\partial x_{k}}-\frac{\partial \bar{u}_{i}}{\partial x_{k}} \bar{S}_{k j}-\frac{\partial \bar{u}_{j}}{\partial x_{k}} \bar{S}_{k i}
\end{gathered}
$$

are, respectively, the mean rate of strain tensor and its frame-indifferent Oldroyd derivative while $C_{D}$ is a dimensionless constant that assumes a value of 1.68 . Of course, the standard eddy viscosity model (1) is recovered in the limit as $C_{D} \rightarrow 0$.

The Reynolds stress models (1) and (4) are solved in conjunction with modeled transport equations for $K$ and $\tau$ which take the form [4]:

$$
\frac{\partial K}{\partial t}+\bar{u}_{i} \frac{\partial K}{\partial x_{i}}=-\tau_{i j} \frac{\partial \bar{u}_{i}}{\partial x_{j}}-\frac{K}{\tau}+\frac{\partial}{\partial x_{i}}\left[\left(v+\frac{v_{T}}{\sigma_{K}}\right) \frac{\partial K}{\partial x_{i}}\right]
$$




$$
\begin{aligned}
\frac{\partial \tau}{\partial t}+\bar{u}_{i} \frac{\partial \tau}{\partial x_{i}}= & \left(C_{\varepsilon 1}-1\right) \frac{\tau}{K} \tau_{i j} \frac{\partial \bar{u}_{i}}{\partial x_{j}}+\left(C_{\varepsilon 2} f_{2}-1\right)+\frac{\partial}{\partial x_{i}}\left[\left(v+\frac{v_{T}}{\sigma_{\tau 2}}\right) \frac{\partial \tau}{\partial x_{i}}\right] \\
& -\frac{2}{\tau}\left(v+\frac{v_{T}}{\sigma_{\tau 2}}\right) \frac{\partial \tau}{\partial x_{i}} \frac{\partial \tau}{\partial x_{i}}+\frac{2}{K}\left(v+\frac{v_{T}}{\sigma_{\tau 1}}\right) \frac{\partial K}{\partial x_{i}} \frac{\partial \tau}{\partial x_{i}}
\end{aligned}
$$

where

$$
f_{2}=\left[1-\frac{2}{9} \exp \left(-\frac{R e_{t}^{2}}{36}\right)\right]\left[1-\exp \left(-\frac{y^{+}}{5}\right)\right]^{2}
$$

is a wall damping function that is needed for asymptotic consistency. The constants $C_{\varepsilon 1}$ and $C_{\varepsilon 2}$ assume the values of 1.44 and 1.83 , respectively, whereas for their preliminary calculations, Speziale et al. [4] chose

$$
\sigma_{\tau 1}=\sigma_{\tau 2}=\sigma_{K}=1.36
$$

with the understanding that these constants could be fine tuned to a non-equivalent set of values when a future optimization over a variety of turbulent flows is conducted. When $\sigma_{\tau 1}, \sigma_{\tau 2}$ and $\sigma_{K}$ are identically equal, this $K-\tau$ model reduces to an equivalent $K$ - $\varepsilon$ model which can be simply obtained by replacing $\tau$ with $K / \varepsilon$ in (7) and replacing the $\tau$-transport equation (8) with the equivalent equation

$$
\frac{\partial \varepsilon}{\partial t}+\bar{u}_{i} \frac{\partial \varepsilon}{\partial x_{i}}=-C_{\varepsilon 1} \frac{\varepsilon}{K} \tau_{i j} \frac{\partial \bar{u}_{i}}{\partial x_{j}}-C_{\varepsilon 2} f_{2} \frac{\varepsilon^{2}}{K}+\frac{\partial}{\partial x_{i}}\left[\left(v+\frac{v_{T}}{\sigma_{\varepsilon}}\right) \frac{\partial \varepsilon}{\partial x_{i}}\right]
$$

where $\sigma_{\varepsilon}$ is equal to the common value of $\sigma_{\tau 1}, \sigma_{\tau 2}$, and $\sigma_{K}$ given in (10).

\section{Discussion of Results}

The first problem that we will consider is the incompressible flat plate boundary layer with adverse pressure gradients - the Samuel and Joubert [9] test case. This represents a rather severe test since the adverse pressure gradient is strong enough so that the flow is not that far removed from separation. In Figure 1(a), the mean velocity profiles predicted by the standard $K$ - $\tau$ model using the eddy-viscosity representation (1) are compared with the experimental data [9] at two stations: $x=1.76 \mathrm{~m}$ and $x=2.87 \mathrm{~m}$ (this corresponds to free-stream Reynolds numbers $R e_{x}$ in the range of $2 \times 10^{6}$ to $4 \times 10^{6}$ ). It is clear from these figures that the model compares quite favorably with the experimental results. In Figure 1(b), the skin friction predicted by the $K-\tau$ model is compared with the experimental data. Again, the agreement between the model predictions and the experimental data is extremely good. Unlike most other two-equation models, the $K$ - $\tau$ model does not overpredict the skin friction for this adverse pressure gradient test case (see, Rodi and Scheuerer [10] for an interesting discussion of this issue). It should be noted that the use of an anisotropic eddy-viscosity does not yield a significant change in the results for this case. 
The second problem to be considered is incompressible turbulent flow past a backward-facing step - the same test case considered by Driver and Seegmiller [11] in their recent experiments. For this flow configuration the aspect ratio $H_{1} / H$ is 8 and the Reynolds number based on the inlet free stream velocity and step height is approximately 38,500 (see Figure 2). The calculations were conducted on a $200 \times 100$ nonuniform mesh using a finite volume method (see Thangam and Hur [12]). Here, we use the anisotropic eddy-viscosity model (4) since it has been recently demonstrated that such models give rise to improved predictions in recirculating flows $[8,12]$. In Figure 3(a), the computed streamlines obtained from the anisotropic $K$ - $\tau$ model are shown. They indicate reattachment at approximately 6 step heights downstream of separation - a result that is in agreement with the experimental data [11]. In Figure 3(b), the mean velocity profiles obtained from the anisotropic $K-\tau$ model are compared with the experimental results; again the agreement is extremely good. In Figure 4(a), the profiles of the turbulence intensity obtained from the anisotropic $K$ - $\tau$ model are compared with the experimental data. While there are some discrepancies between these results in the separation and recovery zone, on balance, the agreement is fairly good. In Figure 4 (b), the profiles of the Reynolds shear stress obtained from the anisotropic $K-\tau$ model are compared with the experimental data [11]. Again, while there are some discrepancies in the results within the separation and recovery zone, the model predictions are still satisfactory. There is no question that this near wall model does a substantially better job than the standard $K$ - $\varepsilon$ model which underpredicts the reattachment point by an amount of the order of $25-30 \%$.

\section{Conclusion}

The near wall $K-\tau$ model of Speziale et al. [4] has been tested in two applications involving incompressible turbulent flows and the results obtained are quite encouraging. The standard $K$ - $\tau$ model - with an isotropic eddy viscosity - yielded excellent results for the challenging incompressible adverse pressure gradient case of Samuel-Joubert [9]. For the separated flow considered - namely, turbulent flow past a backward facing step - excellent results were obtained from the $K-\tau$ model based on an anisotropic eddy-viscosity. In fact, we believe that these may be the most accurate calculations for this backstep problem that have yet been obtained from a two-equation model without the ad hoc adjustment of constants.

Future calculations are planned for supersonic boundary layers with streamline curvature and separation (shock-induced or otherwise). For these flows, the $K$ - $\tau$ model will most probably have to be applied with the anisotropic eddy viscosity model and it may become necessary to incorporate

dilatational effects into the model. After these calculations are completed, and refinements are made accordingly, we will have a much better idea of the range of applicability of the $K-\tau$ model. Nonetheless, at this point the prospects appear to be quite promising. 


\section{References}

[1] Launder, B.E., "Phenomenological Modeling: Present and Future," Proc. Whither Turbulence Workshop (ed. J.L. Lumley), Lecture Notes in Physics, Vol. 357, pp. 439-485. Springer-Verlag, Berlin, 1990.

[2] Speziale, C.G., "Analytical Methods for the Development of Reynolds Stress Closures in Turbulence," Ann. Rev. of Fluid Mech., Vol. 23, pp. 107-157, 1991.

[3] Patel, V.C., Rodi, W., and Scheuerer, G., "Turbulence Models for Near-Wall and Low Reynolds Number Flows: A Review," AIAA J., Vol. 23, pp. 1308-1319, 1985.

[4] Speziale, C.G., Abid, R., and Anderson, E.C., "A Critical Evaluation of Two-Equation Models for Near Wall Turbulence," AIAA Paper 90-1481, 1990.

[5] Reynolds, W.C., "Fundamentals of Turbulence for Turbulence Modeling and Simulation," Lecture Notes for Von Karman Institute, AGARD Lecture Series, No. 86, pp. 1-66. NATO, New York, 1987.

[6] Zeierman, S. and Wolfshtein, M., "Turbulent Time Scale for Turbulent Flow Calculations," AIAA J., Vol. 24, pp. 1606-1610, 1986.

[7] Yoshizawa, A., "Statistical Analysis of the Deviation of the Reynolds Stress from its Eddy Viscosity Representation," Phys. Fluids, Vol. 27, pp. 1377-1389, 1984.

[8] Speziale, C.G., "On Nonlinear $K-l$ and $K-\varepsilon$ Models of Turbulence," J. Fluid Mech., Vol. 178, pp. 459-475, 1987.

[9] Samuel, A.E. and Joubert, P.N., "A Boundary Layer Developing in an Increasing Adverse Pressure Gradient," J. Fluid Mech., Vol. 66, pp. 481-493, 1974.

[10] Rodi, W. and Scheuerer, G., "Scrutinizing the $K-\varepsilon$ Turbulence Model Under Adverse Pressure Gradient Conditions," ASME J. Fluids Engrg., Vol. 108, pp. 174-179, 1986.

[11] Driver, D.M. and Seegmiller, H.L., "Features of a Reattaching Turbulent Shear Layer in Divergent Channel Flow," AIAA J., Vol. 23, pp. 163-171, 1985.

[12] Thangam, S. and Hur, N., "A Highly Resolved Numerical Study of Separated Flow Past a Backward Facing Step," Int. J. Engrg Sci. (in press). 

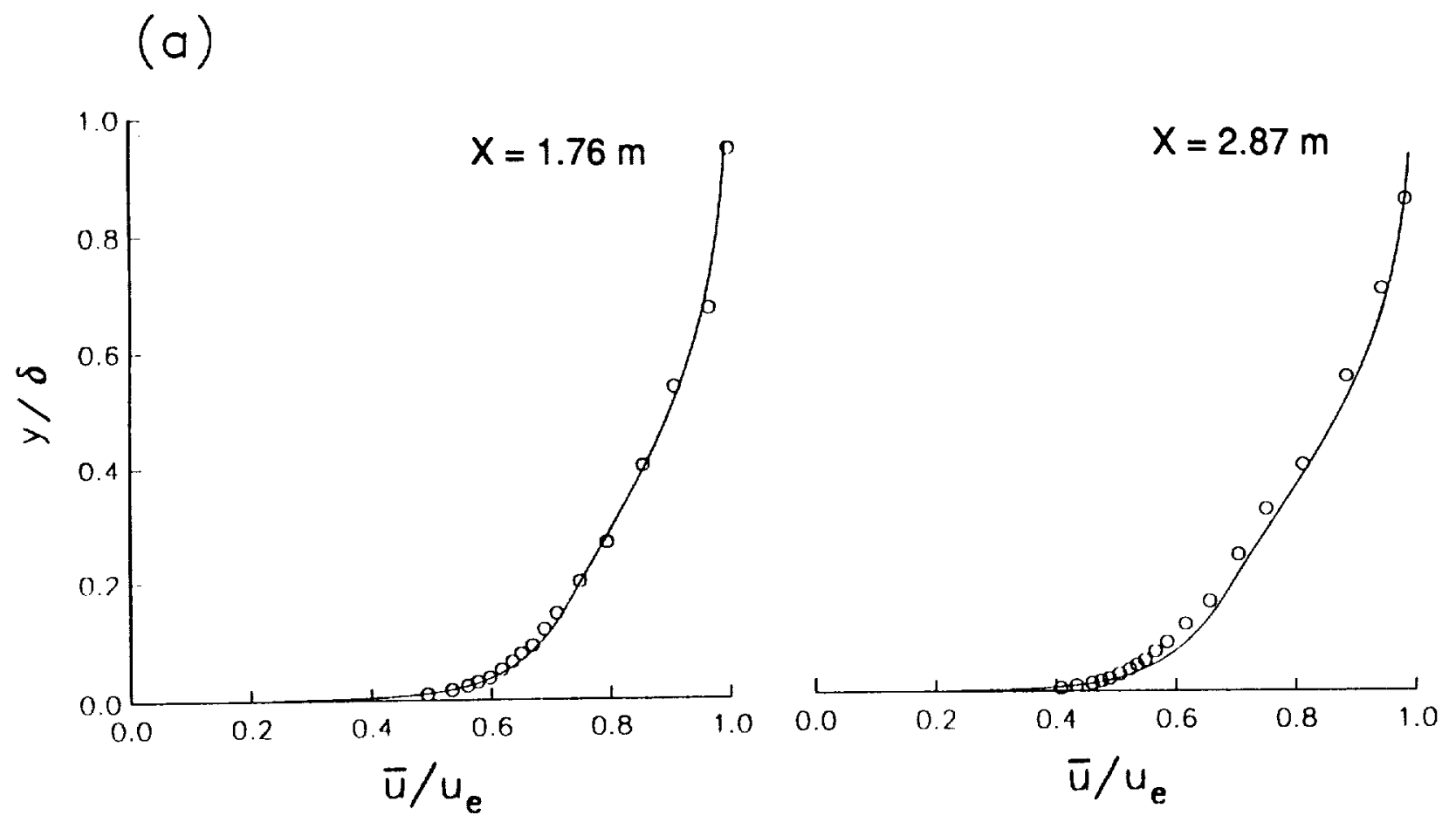

(b)

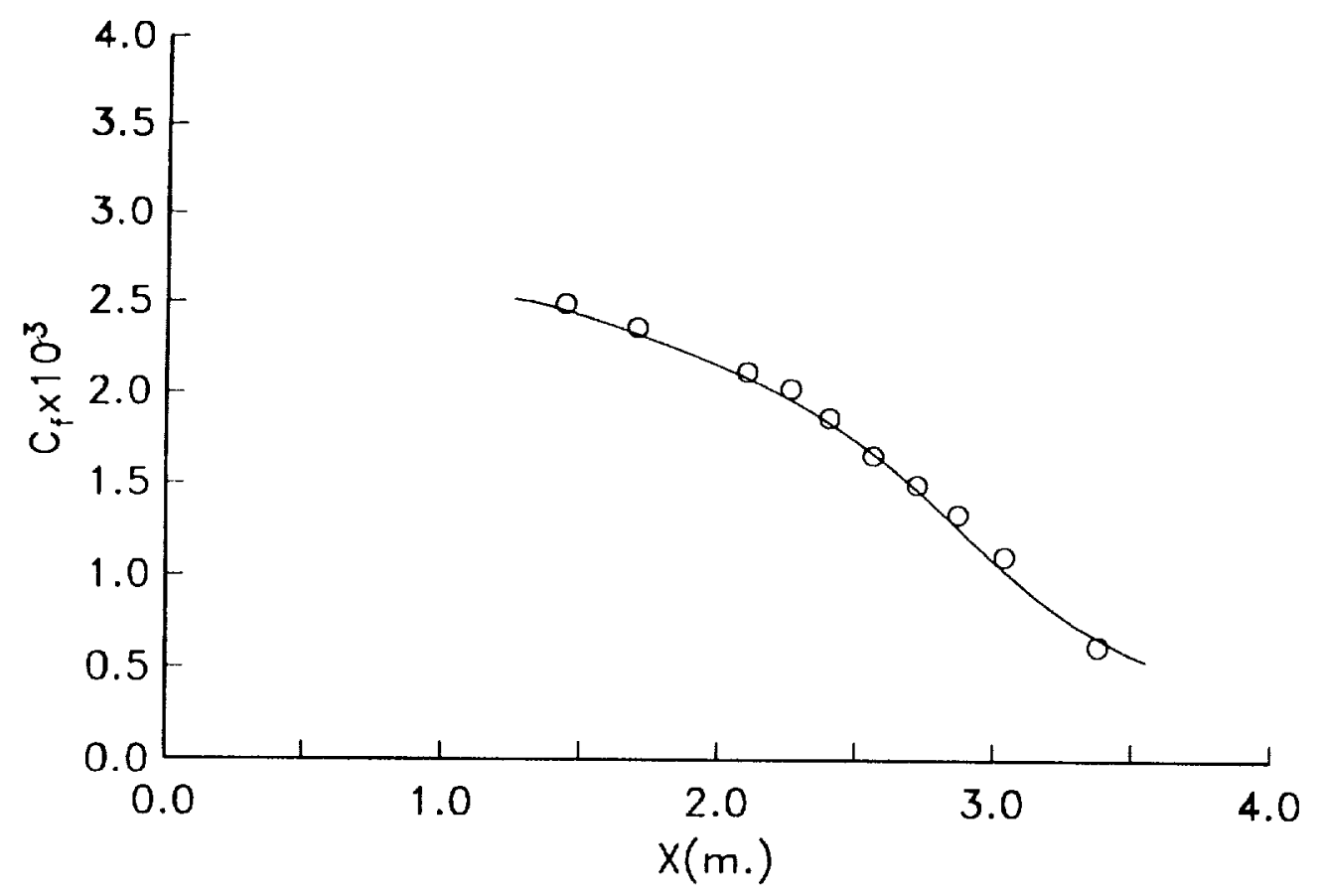

Figure 1. Comparison of the results of the $K-\tau$ Model and experiments for the flat plate boundary layer with an adverse pressure gradient $(-K-\tau$ Model, o experiments of Samuel \& Joubert [9]) :

(a) mean velocity, and (b) skin friction coefficient 


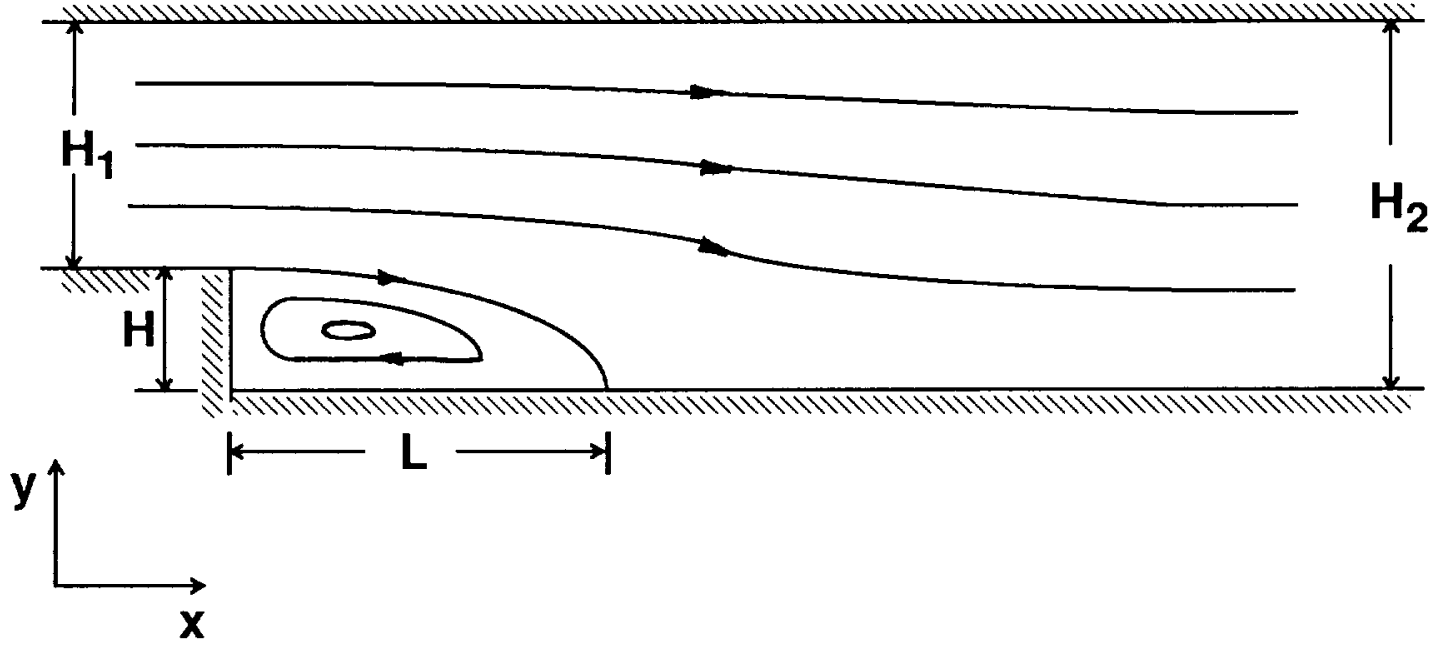

Figure 2. Schematic of the diagram for turbulent flow past a backward facing step 
(a)

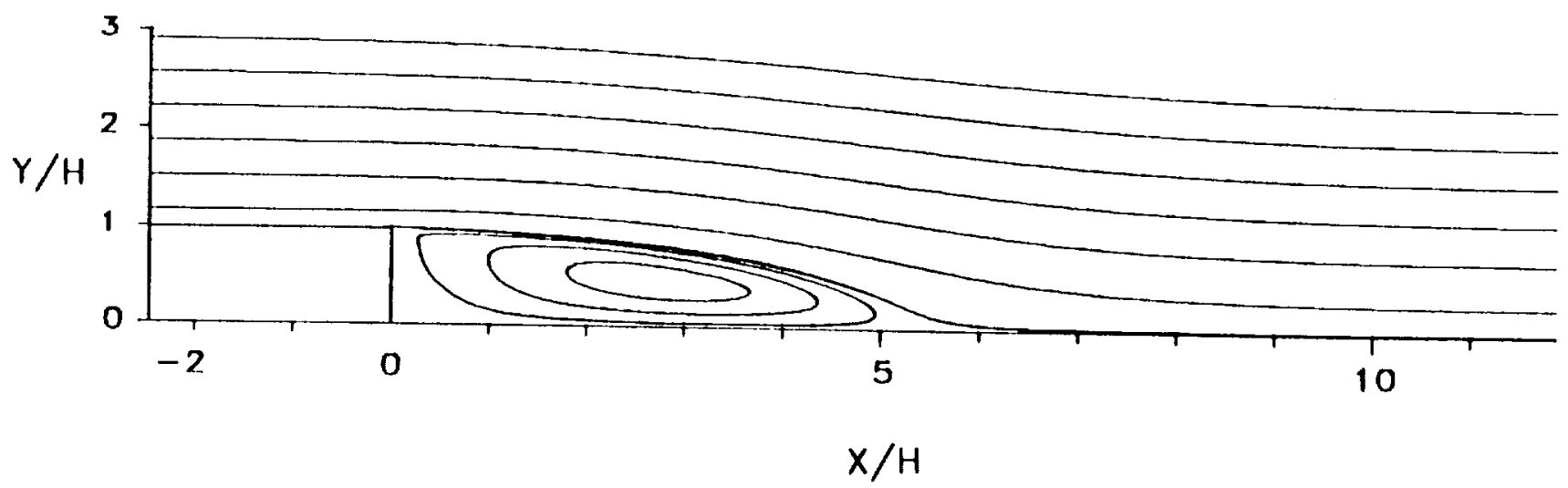

(b)

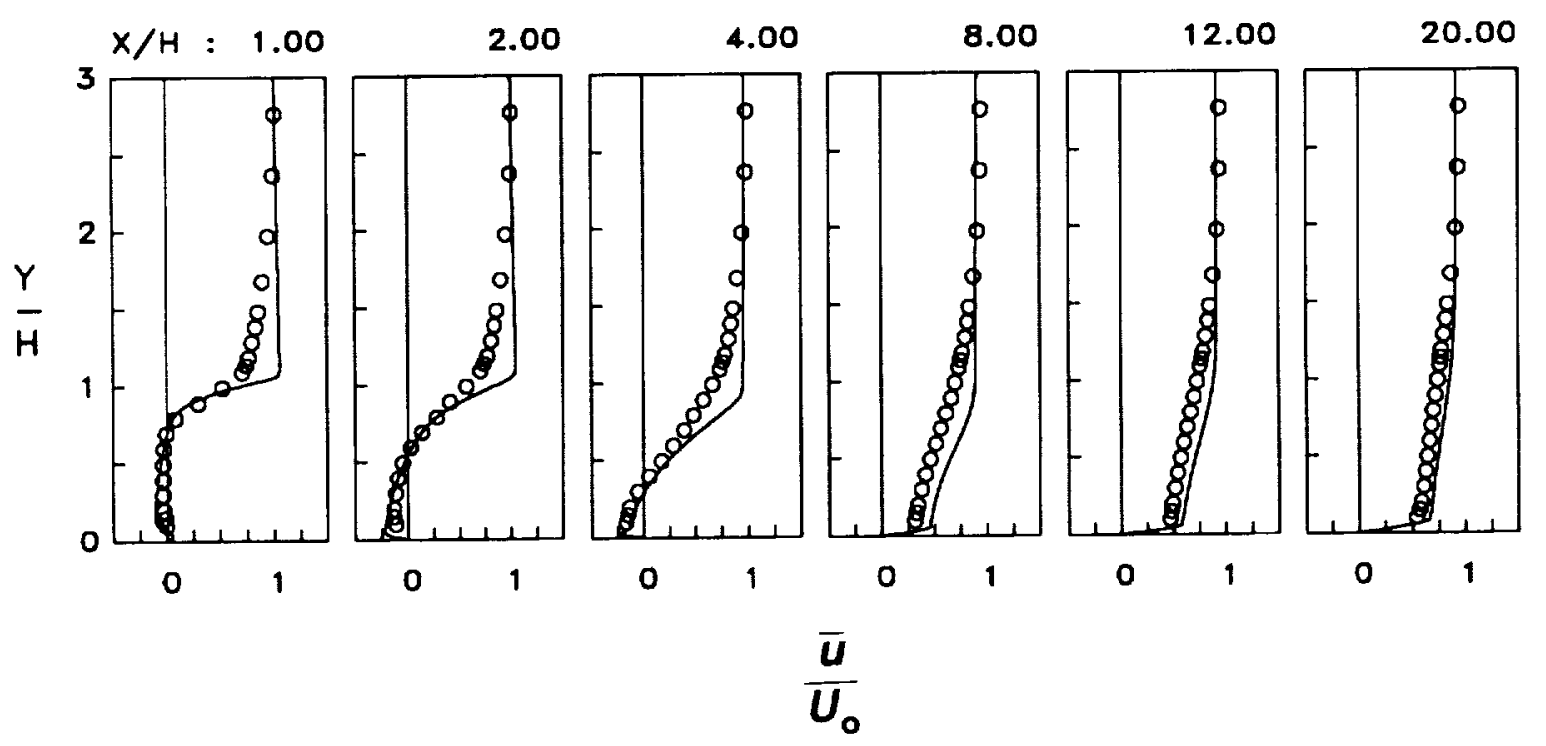

Figure 3. Results obtained from the anisotropic $K-\tau$ Model for incompressible turbulent flow past a backward facing step with $\mathrm{H}_{1} / \mathrm{H}=8, R e=38,500$. ( $-K-\tau$ Model, o experiments of Driver \& Seegmiller [11]):

(a) mean velocity streamlines, and (b) mean velocity profiles. 
(a)

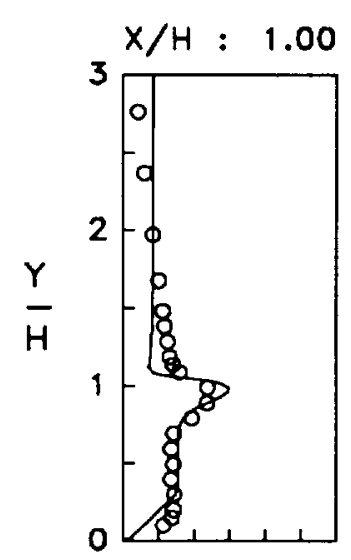

$0.0 \quad 0.10 .2$

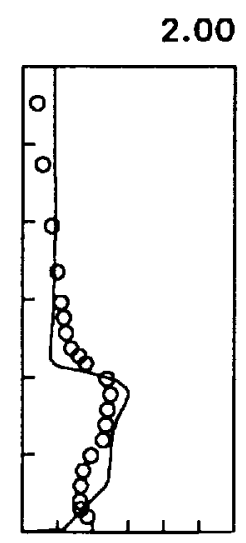

0.10 .2

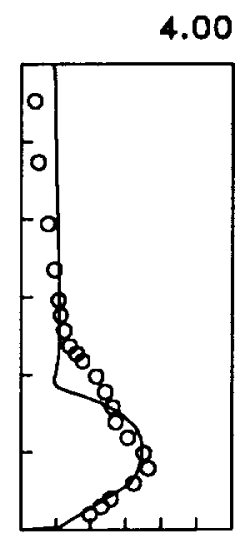

0.10 .2
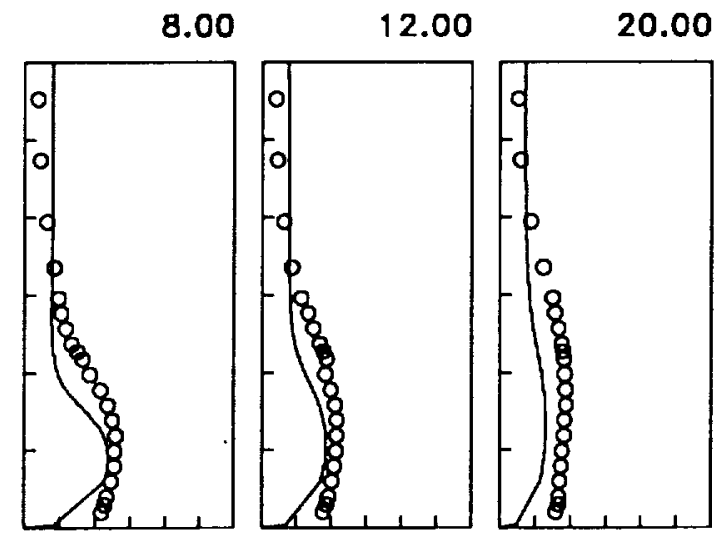

0.10 .2

0.10 .2

0.10 .2

$$
\left.(\overline{u u})^{2}\right)^{1 / 2}
$$

(b)

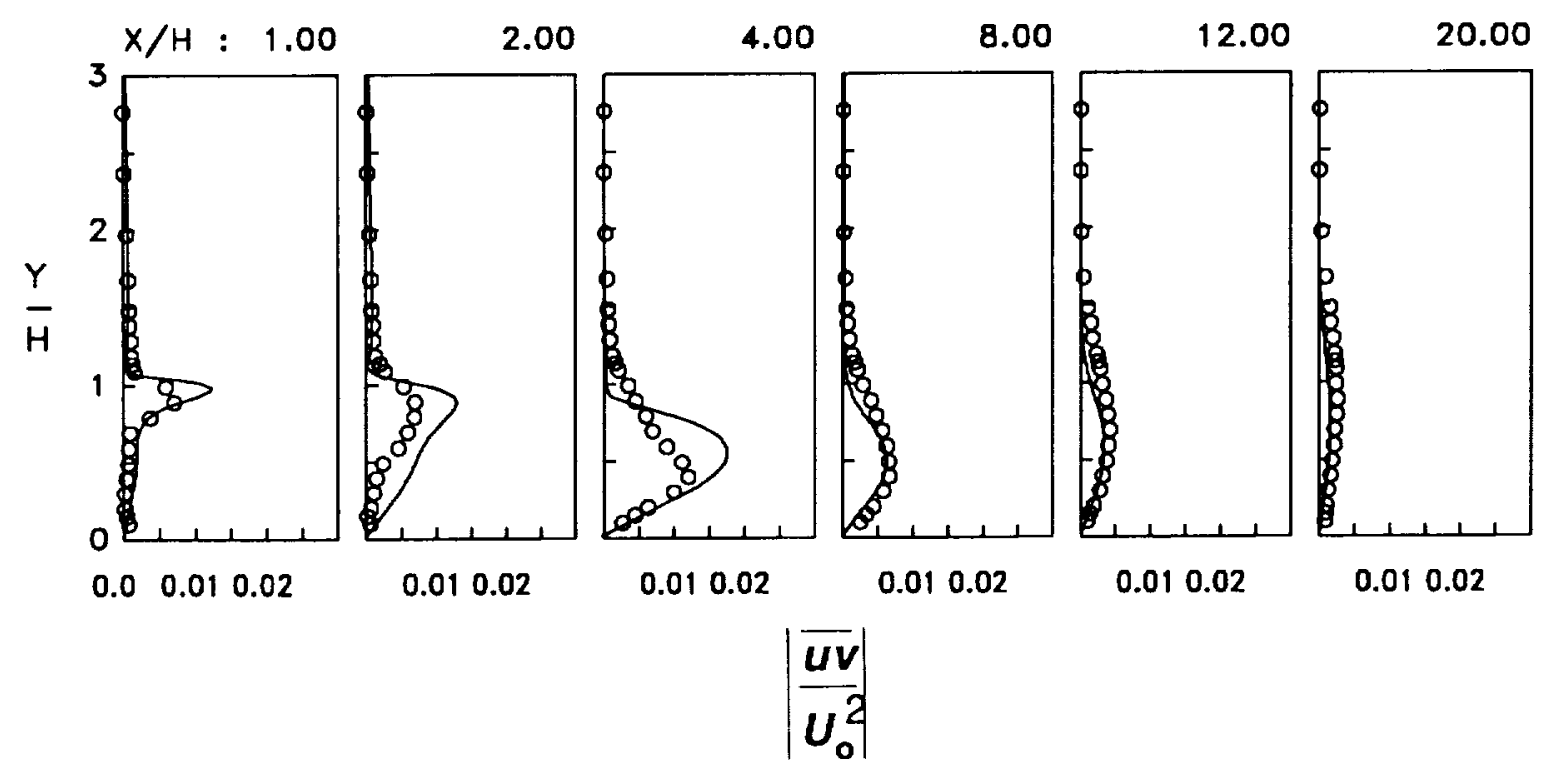

Figure 4. Results obtained from the anisotropic $K-\tau$ Model for incompressible turbulent flow past a backward facing step with $\mathrm{H}_{1} / \mathrm{H}=8, R e \approx 38,500$. ( $-K-\tau$ Model, o experiments of Driver \& Seegmiller [11]):

(a) turbulence intensity profiles, and (b) Reynolds stress profiles. 


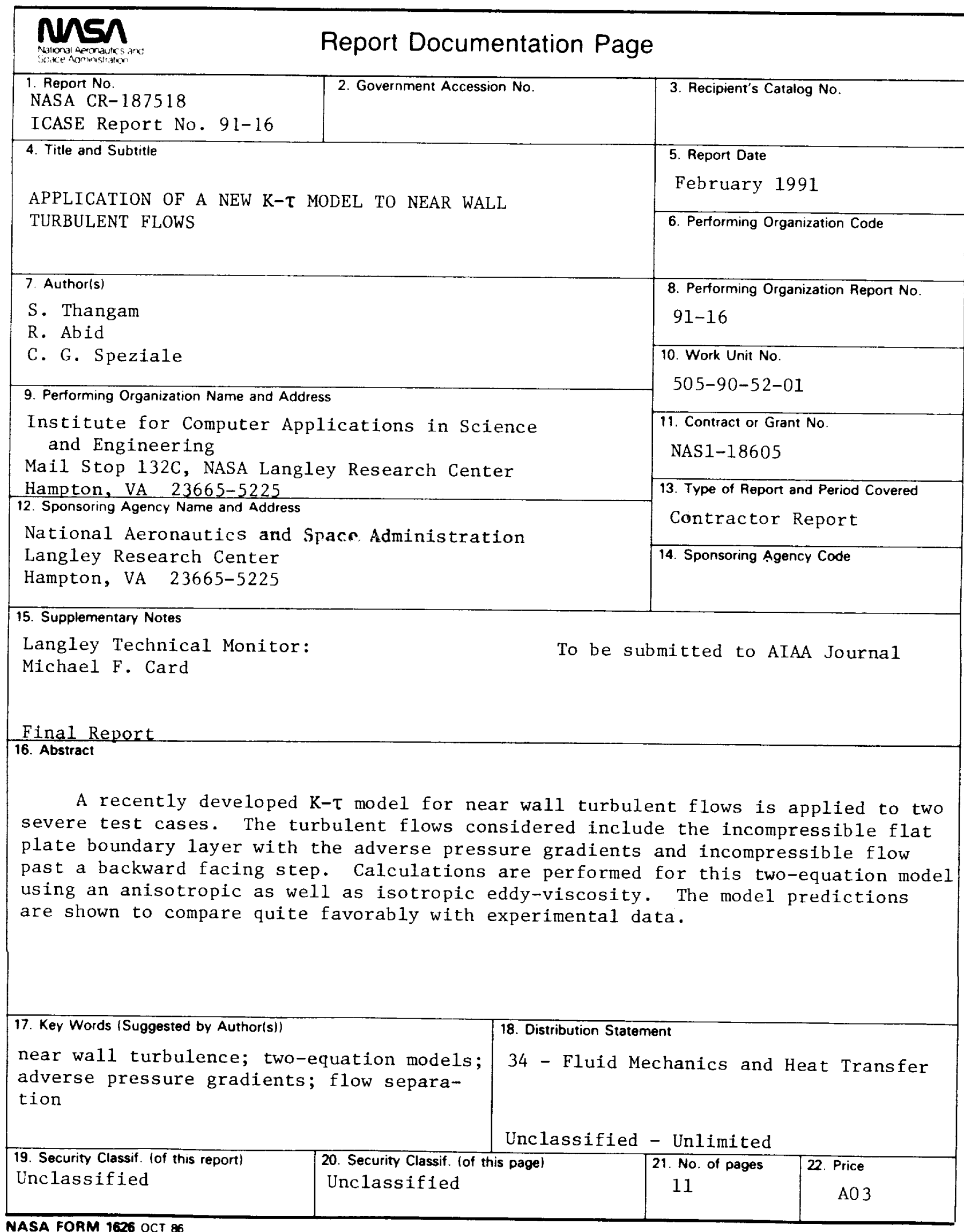



